\title{
MaxEnt Alternatives to Pearson Family Distributions
}

\author{
Barrie J Stokes \\ Discipline of Clinical Pharmocolgy, University of Newcastle, Australia
}

\begin{abstract}
In a previous MaxEnt conference [11] a method of obtaining MaxEnt univariate distributions under a variety of constraints was presented. The Mathematica function Interpolation[], normally used with numerical data, can also process "semi-symbolic" data, and Lagrange Multiplier equations were solved for a set of symbolic ordinates describing the required MaxEnt probability density function. We apply a more developed version of this approach to finding MaxEnt distributions having prescribed $\beta_{1}$ and $\beta_{2}$ values, and compare the entropy of the MaxEnt distribution to that of the Pearson family distribution having the same $\beta_{1}$ and $\beta_{2}$. These MaxEnt distributions do have, in general, greater entropy than the related Pearson distribution. In accordance with Jaynes' Maximum Entropy Principle, these MaxEnt distributions are thus to be preferred to the corresponding Pearson distributions as priors in Bayes' Theorem.
\end{abstract}

Keywords: maximum entropy distributions, Pearson distributions, Mathematica

PACS: 89.70.Cf

\section{INTRODUCTION}

Karl Pearson introduced his eponymous family of distributions in an Abstract [1] and the three papers [2], [3], and [4]. To quote from the comprehensive Wikipedia article [6]:

"The Pearson system was originally devised in an effort to model visibly skewed observations. It was well known at the time how to adjust a theoretical model to fit the first two cumulants or moments of observed data: Any probability distribution can be extended straightforwardly to form a location-scale family. Except in pathological cases, a location-scale family can be made to fit the observed mean (first cumulant) and variance (second cumulant) arbitrarily well. However, it was not known how to construct probability distributions in which the skewness (standardized third cumulant) and kurtosis (standardized fourth cumulant) could be adjusted equally freely. This need became apparent when trying to fit known theoretical models to observed data that exhibited skewness. Pearson's examples include survival data, which are usually asymmetric."

The generality of the resulting Pearson family is quite astounding. Many familiar (and not so familiar) distributions are in fact Pearson distributions, including the Arcsine, Beta, Beta Prime, Cauchy, Chi-Square, Erlang, Exponential, F Ratio, Gamma, Half Normal, Hotelling's $T^{2}$, Inverse Chi-Square, Inverse Gamma, Levy, Normal, Pareto, Power, Student t, and Wigner Semicircle distributions.

Bayesian Inference and Maximum Entropy Methods in Science and Engineering

AIP Conf. Proc. 1443, 250-259 (2012); doi: 10.1063/1.3703642

(C) 2012 American Institute of Physics 978-0-7354-1039-8/\$30.00 
In Section 1 we show how Pearson derived a differential equation for a continuous PDF $p(x)$ based on the "relative slope" of the BinomialDistribution[n,p] with $p=1 / 2$. This differential equation takes the form

$$
\frac{d p(x)}{\mathrm{dx}}=-(a x+b) p(x)
$$

In Section 2 we demonstrate that the same approach to the Hypergeometric distribution yields a more general differential equation of the form,

$$
\frac{d p(x)}{\mathrm{dx}}=-\frac{(a x+b)}{c x^{2}+d x+e} p(x) .
$$

The potential here recognised by Pearson is that:

1. the point of zero slope at $x=-b / a$, i.e., the mode of the PDF defined by $p(x)$, is independent of the roots of the quadratic form in the denominator, so that a variety of skewed distributions can be realized; and

2. depending on the nature of the roots (both real and same sign, both real and opposite sign, both complex), the resulting distribution can be in the classes that Pearson called Type VI, Type I, or Type IV respectively.

It is the variety of combinations of possible parameter values, and the consequent positions of the zero in the numerator and the roots of the denominator, that give rise to the richness of the Pearson distribution system,

Since the critical quantity governing the nature of the roots of $c x^{2}+d x+e$ is $d^{2} / 4 c e$, Pearson derived this quantity in terms of the moments $\beta 1$ (skewness) and $\beta 2$ (kurtosis) as

$$
\kappa=\frac{\beta_{1}\left(3+\beta_{2}\right)^{2}}{4\left(3 \beta_{1}-4 \beta_{2}\right)\left(6+3 \beta_{1}-2 \beta_{2}\right)}
$$

If $\kappa<0$, we have a Type I distribution, if $0<\kappa<1$ we have Type IV, and if $\kappa>1$ we have Type VI. There are also a couple of "transition" Types for $\kappa=0$ and $\kappa=1$, among which are the Gaussian and the Gamma distributions.

In Section 3 Rhind's [7] $\beta 1-\beta 2$ plane is introduced, and in Section 4 our approach to constructing MaxEnt alternatives to Pearson distributions is briefly described. Section 5 then shows some MaxEnt alternatives to several Pearson distribution Types, as discussed in [8].

\section{THE SYMMETRIC BINOMIAL}

We proceed as Pearson did, by first considering the Binomial distribution with $p=1 / 2$. We set up a function for this Binomial PMF (Probability Mass Function) ...

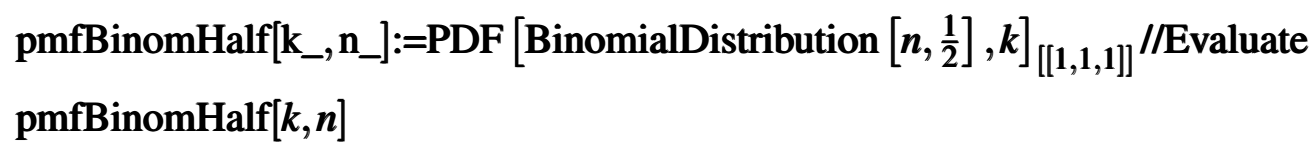


$2^{-n} \operatorname{Binomial}[n, k]$

This discrete distribution is both symmetric and quite Gaussian in general shape ( $\mathrm{n}=$ 30), as shown in Fig. 1.

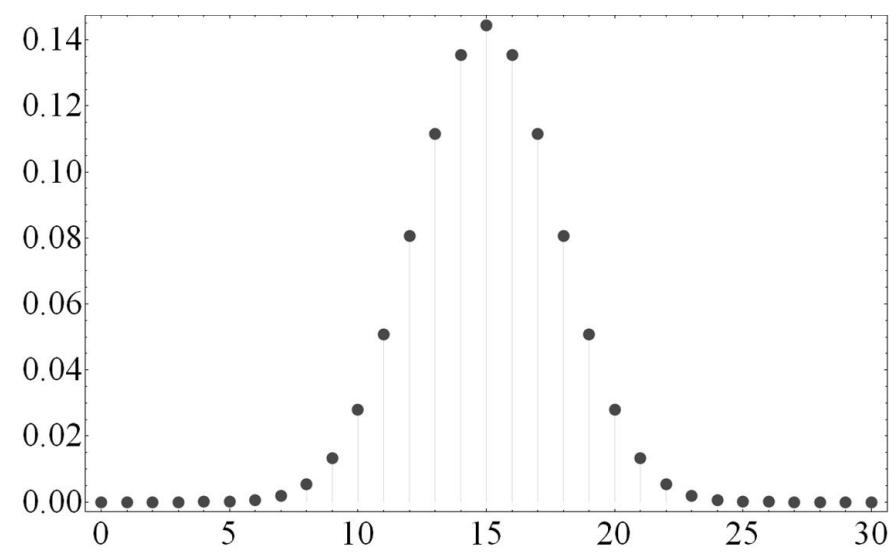

FIGURE 1. PMF[BinomialDistribution[30,1/2],k]

Pearson noted that the relative slope (here we use the "central relative slope") of these Binomial probabilities ...

centralRelativeSlope $=\frac{\text { pmfBinomHalf }[k+1 / 2, n]-\text { pmfBinomHalf }[k-1 / 2, n]}{\frac{1}{2}(\operatorname{pmfBinomHalf}[k+1 / 2, n]+\text { pmfBinomHalf }[k-1 / 2, n])} / /$ FullSimplify $/ /$

\section{Together}

$\frac{2(-2 k+n)}{1+n}$

$\ldots$ it is clearly linear in $\mathrm{k}$ with negative slope. Solving the differential equation $\mathrm{p}^{\prime}(\mathrm{x}) / \mathrm{p}(\mathrm{x})=$ centralRelativeSlope, we get:

sol1 $=$ DSolve $\left[\frac{p^{\prime}[k]}{p[k]}==\text { centralRelativeSlope }, p[k], k\right]_{[[1,1]]}$

$p[k] \rightarrow e^{\frac{2\left(-k^{2}+k n\right)}{1+n}} C[1]$

The consequent (Gaussian) PDF is

$$
\exp \left(-\frac{2}{31}(k-15)^{2}\right) \sqrt{\frac{2}{31 \pi}},
$$

and the two distributions each have mean $=15$, and compare as in Figs. 2 and 3.

\section{THE HYPERGEOMETRIC DISTRIBUTION}

A hypergeometric distribution gives the distribution of the number of successes in $n$ draws from a population of size $n_{\text {tot }}$ containing $n_{\text {succ }}$ successes.

Here we set up a function for the Hypergeometric PDF with success probability of 30/100: 


\begin{tabular}{l|ll|}
\hline & \multicolumn{1}{|c|}{$\begin{array}{c}\text { discrete } \\
\text { Binomial Distribution }\end{array}$} & $\begin{array}{l}\text { continuous } \\
\text { diff. eqn. solution }\end{array}$ \\
\hline total probabilities & 1. & 1 \\
means & 15. & 15. \\
variances & 7.74998 & 7.5 \\
skewnesses & $1.15064 \times 10^{-6}$ & 0. \\
kurtoses & 2.99994 & 2.93333 \\
\hline
\end{tabular}

FIGURE 2.

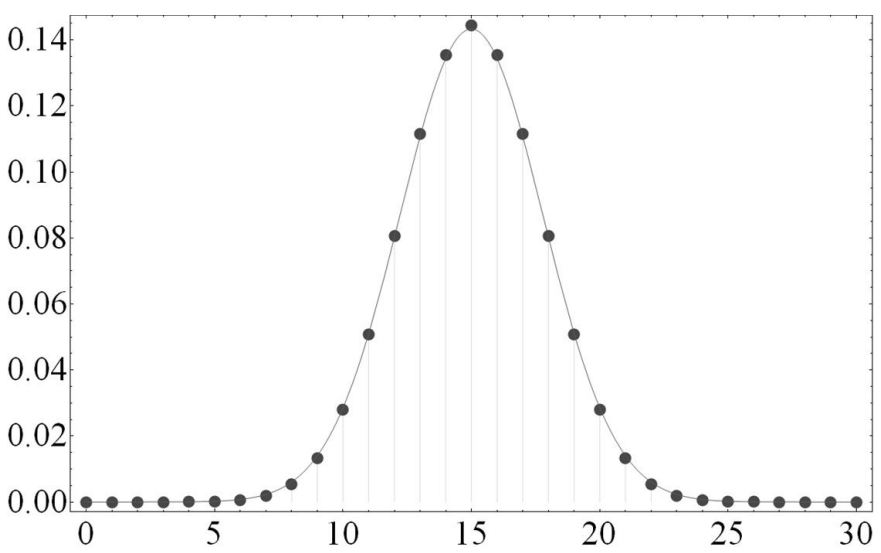

FIGURE 3. PMF[BinomialDistribution[30,1/2],k] and PDF of corresponding Pearson Distribution (central relative slope)

pmfHypergeometricPointThree[k_, $\left.\mathbf{n}_{-}\right]:=$

$\left.\operatorname{PDF}[\text { HypergeometricDistribution }[n, 30,100], k]_{[[1,1,1]]}\right] /$ Evaluate

pmfHypergeometricPointThree $[k, n] / /$ TraditionalForm

$\frac{\left(\begin{array}{c}30 \\ k\end{array}\right)\left(\begin{array}{c}70 \\ n-k\end{array}\right)}{\left(\begin{array}{c}100 \\ n\end{array}\right)}$

This probability mass function for $\mathrm{n}=15$ draws is depicted in Fig. 4 .

The central relative slope of these pmfHypergeometricPointThree probabilities is ...

centralRelativeSlope $=$

(pmfHypergeometricPointThree $[k+1 / 2,15]-$ pmfHypergeometricPointThree[ $k-1 / 2,15]) /$

$\left(\frac{1}{2}(\right.$ pmfHypergeometricPointThree $[k+1 / 2,15]+$ pmfHypergeometricPointThree $\left.[k-1 / 2,15])\right) / /$

FullSimplify//Together

$-\frac{4(-445+102 k)}{1001+20 k+4 k^{2}}$ 


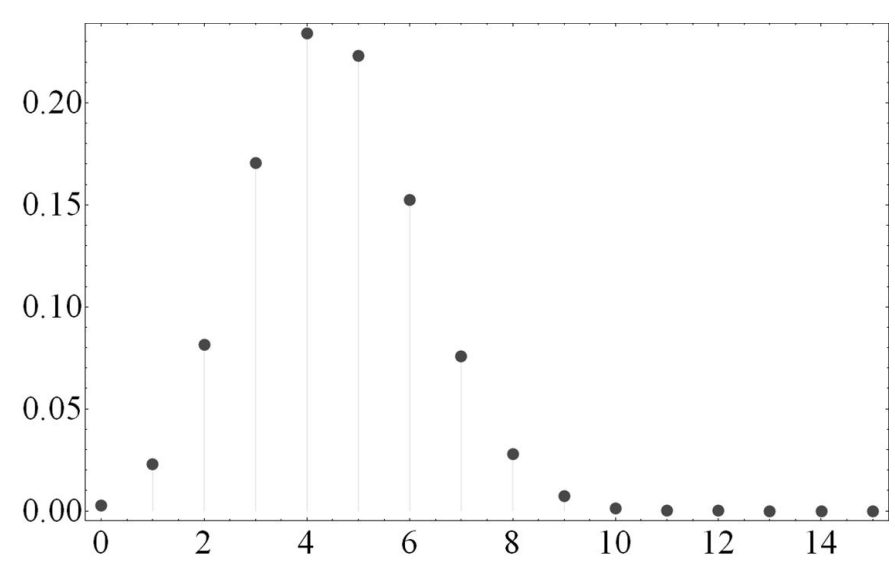

FIGURE 4. PMF[HyperGeometricDistribution[15,30,100],k]

... and is also linear in $\mathrm{k}$ with negative slope in the numerator, but now with a quadratic denominator in $\mathrm{k}$. Solving the differential equation $\mathrm{p}^{\prime}(\mathrm{x}) / \mathrm{p}(\mathrm{x})=$ centralrelativeSlope, and determining the value of the constant of integration via numerical integration, the resulting pdf is

$$
\frac{4.83445 \times 10^{147} e^{\frac{350 \tan ^{-1}\left(\frac{2 k+5}{4 \sqrt{61}}\right)}{\sqrt{61}}}}{\left(4 k^{2}+20 k+1001\right)^{51}} .
$$

We see that this is a distinctly non-standard density (it's a Pearson Type IV), but it has the non-zero skewness that Pearson was trying to encapsulate in his family of distributions. The discrete and continuous distributions compare as in Figs. 5 and 6.

\begin{tabular}{l|ll|} 
& \multicolumn{1}{|c|}{ discrete } & $\begin{array}{l}\text { continuous } \\
\text { diff. eqn. solution }\end{array}$ \\
\hline total probabilities & 0.997159 & 1 \\
means & 4.50131 & 4.5 \\
variances & 2.88903 & 2.70455 \\
skewnesses & 0.242228 & 0.173734 \\
kurtoses & 3.03921 & 2.91701 \\
\hline
\end{tabular}

FIGURE 5.

\section{THE $\beta 1$ - $\beta 2$ PLANE}

Pearson's key idea was that since the "linear divided by quadratic" form of the Pearson differential equation involves 5 parameters, one could fix these coefficients - and thus specify a PDF-if the mean, variance, skewness $\left(\beta_{1}\right)$ and kurtosis $\left(\beta_{2}\right)$ of the target distribution are specified (together with the default condition that the total probability is unity). 


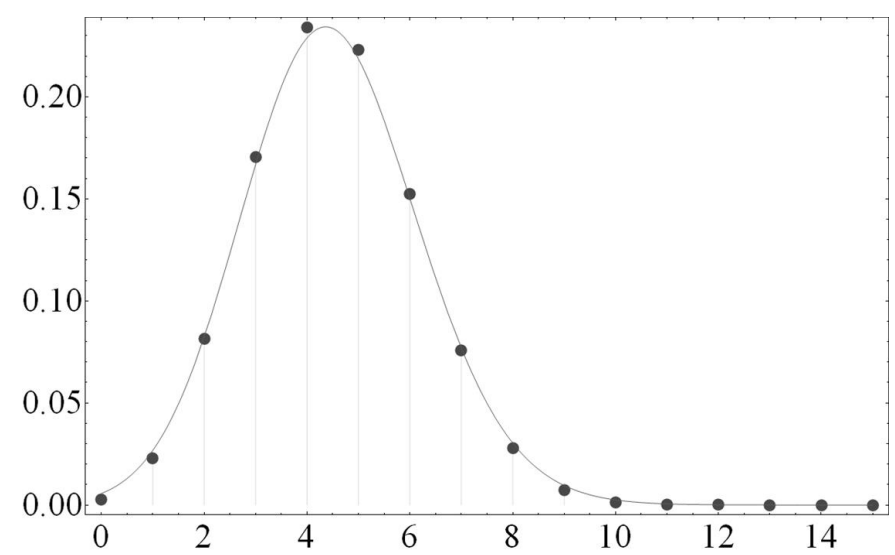

FIGURE 6. PMF[HypergeometricDistribution[15,30,100],k] and PDF of corresponding Pearson Distribution (central relative slope)

Rhind [7] introduced the idea that the Pearson Family of distributions could be visualized on a $\beta_{1}-\beta_{2}$ plane. Rhind's original diagram had the $\beta_{2}$ axis inverted; we will use a more conventional version, showing all XII Types as listed in [8].

\section{MAXENT ALTERNATIVES TO PEARSON FAMILY DISTRIBUTIONS}

The four required moments can be calculated from data to which a summarising Pearson distribution is to be fitted, or they could be specified by a domain expert during an interactive process of Bayesian prior elicitation. In any case, if the four moments were to be used to specify a Bayesian prior distribution in the form of a Pearson distribution, we suggest that a MaxEnt Bayesian prior distribution fitted to the moments can be constructed to serve as the appropriate prior distribution. Why should we do this?

According to Jaynes' principle of Maximum Entropy, if we want to specify a prior distribution in the light of some testable information, then: "Out of all the possible probability distributions which agree with the given constraint information, select the one that is maximally non-committal with regard to missing information." [9] The MaxEnt principle is that the maximally non-committal distribution is the distribution with maximum entropy. Many analytic forms of both discrete and continuous MaxEnt distributions can be found by application of the Lagrange multiplier method [10].

Using the technique reported in [11], standardised MaxEnt distributions corresponding to a variety of standardised Pearson family distributions can be constructed. The correspondence is via the constraining of the MaxEnt distribution by the same $\beta_{1}$ and $\beta_{2}$ specifying the Pearson distribution. By virtue of their manner of construction, these MaxEnt distributions do not have poles at the end of their domains, even when corresponding, for example, to a $\operatorname{Beta}(1 / 2,1 / 2)$ "U-shaped" Pearson distribution of Type I. In some cases the entropy of the MaxEnt distribution is only slightly higher than 
that of the Pearson distribution with the same $\beta_{1}$ and $\beta_{2}$, but there are cases when the difference is several percentage points.

These constructed MaxEnt distributions are in the form of Mathematica InterpolationFunction objects, and can be of linear, quadratic or even higher order if required. Most importantly, the InterpolationFunction form can be manipulated (plotted, differentiated, combined as priors with likelihood functions to generate posteriors) in just the same ways as can standard functions.

\section{MAXENT ALTERNATIVES TO EXAMPLES OF PEARSON TYPES I TO XII}

Using the technique illustrated above, we determine a MaxEnt distribution as an alternative to a given Pearson family distribution by first creating symbolic expressions involving the $\operatorname{ord}_{i}$ - like areaSymbolic and secondMomentSymbolic above - for the entropy (the objective function), the area (set to one), first moment (set to zero), second moment (set to one), and the third and fourth moments as specified by $\beta 1$ and $\beta 2$ for the given Pearson family distribution. Solving for the $\operatorname{ord}_{i}$ and five multipliers using the Lagrange Multiplier method, with entropy as the objective function and these five constraints, using the Mathematica function FindRoot[], a MaxEnt distribution is then determined.

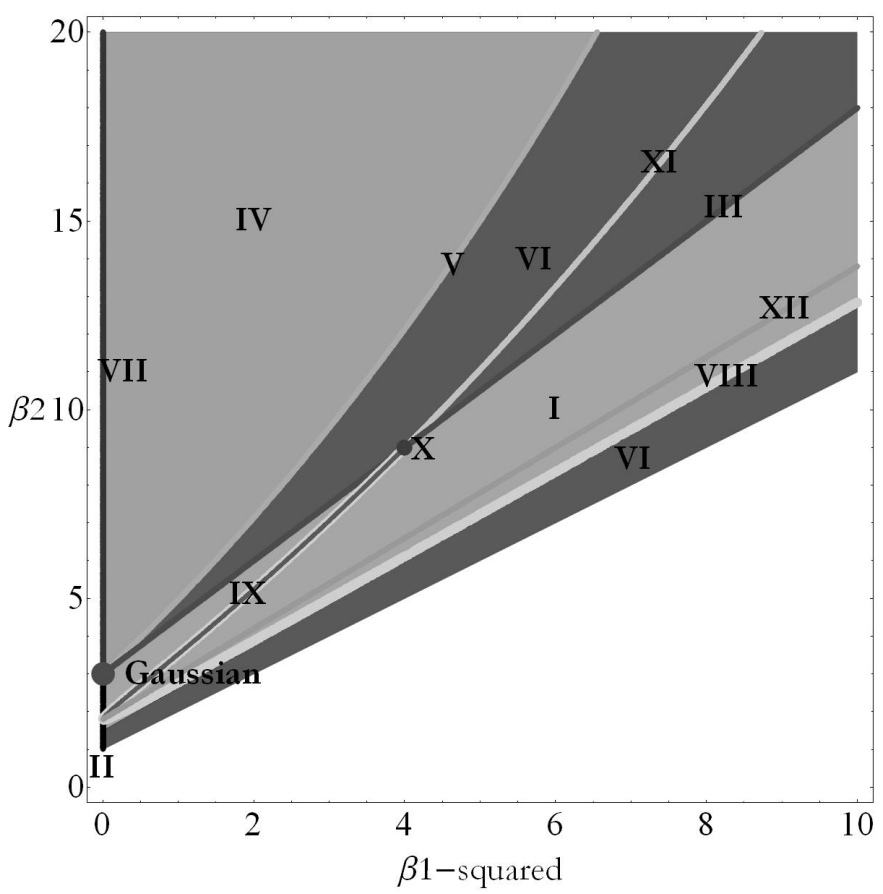

FIGURE 7. Pearson Diagram (after Rhind, with conventional $\beta 2$ axis) showing positions of twelve Pearson Type examples 
Figures 8 through 11 show some examples of Pearson distributions, with their corresponding MaxEnt distributions, contrasting their entropies in each case.

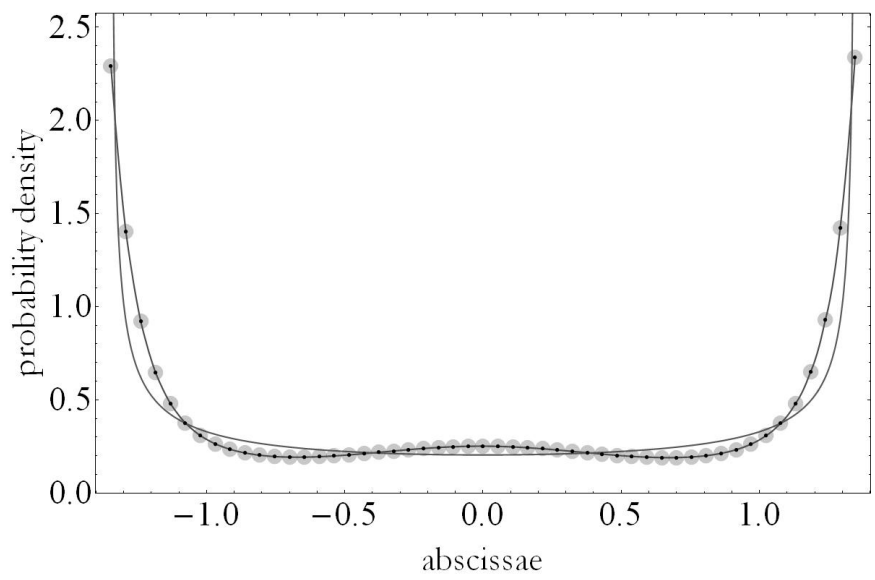

FIGURE 8. A Maxent alternative for a Pearson Type II distribution

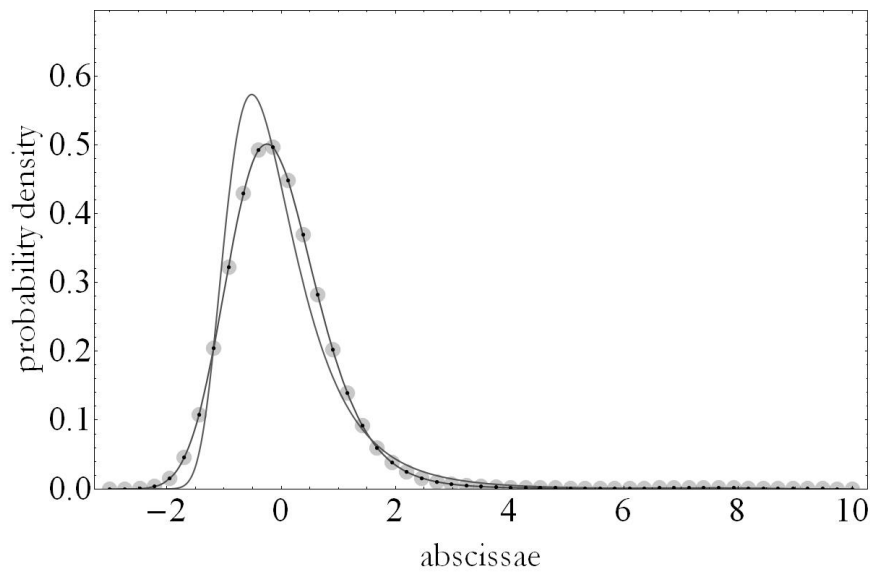

FIGURE 9. A Maxent alternative for a Pearson Type IV distribution

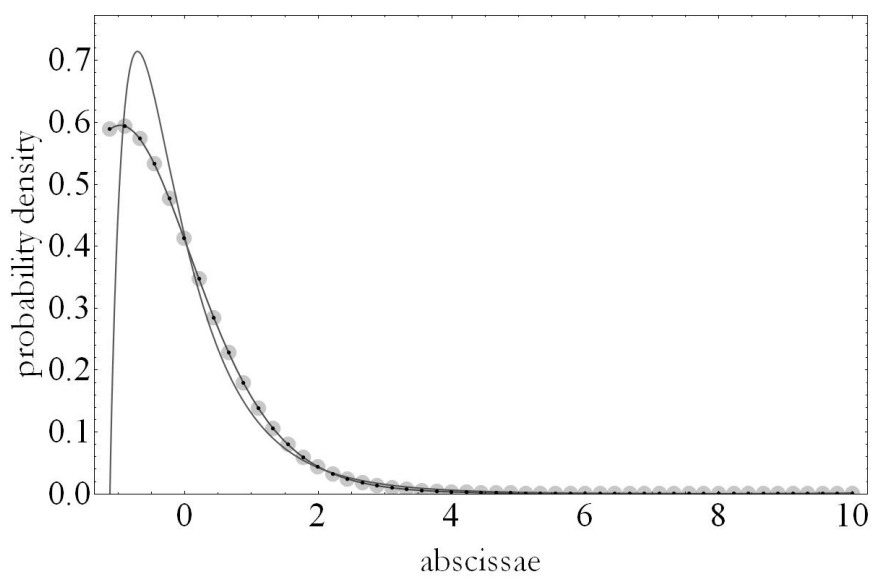

FIGURE 10. A Maxent alternative for a Pearson Type VI distribution 


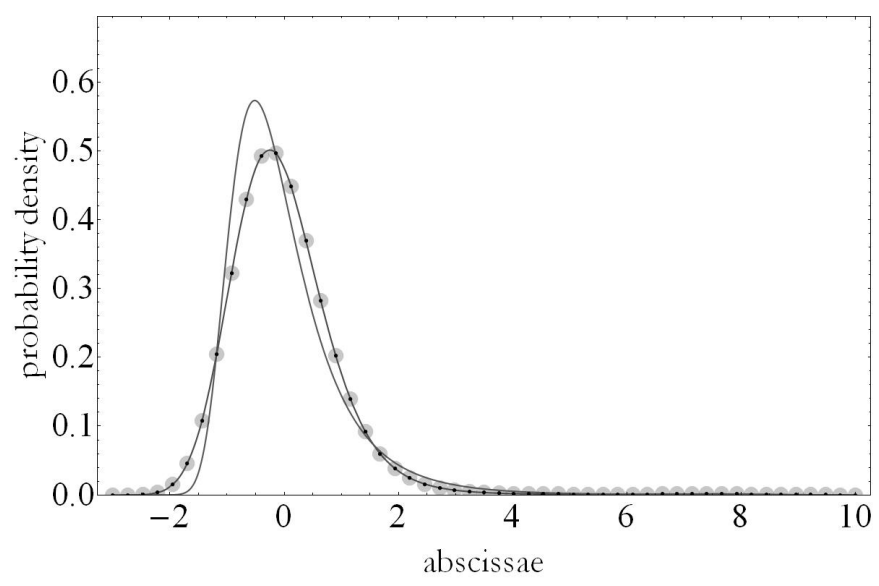

FIGURE 11. A Maxent alternative for a Pearson Type IX distribution

\section{DISCUSSION.}

It is possible to construct distributions-represented by InterpolationFunction objectsmatching the first four moments of a Pearson Distribution while having generally greater entropy. While it appears that in the cases of Types IX, X, and XI the Pearson distribution has an entropy as large as that of the constructed MaxEnt distribution and is similarly shaped, in other cases it is clear that the constructed MaxEnt distribution has larger entropy, and a somewhat different shape than that of the corresponding Pearson distribution.

In the case of the example of Type $\mathrm{V}$ the Pearson distribution has an entropy around 6.5\% lower than that of the MaxEnt distribution with the same first four moments.

According to Jaynes' MaxEnt principle, the constructed MaxEnt distribution would be preferred as a prior to the corresponding Pearson distribution, given the relevant moment constraints.

It would appear that the MaxEnt distribution construction method illustrated here and in [11] is sufficiently flexible to accommodate a variety of constraint types (moments, tail areas), and so could be useful in situations where there would no obvious analytic form of a prior meeting these or more unorthodox constraint types, and for which entropy is maximised.

Future work will examine further constraint types, and the extension of this MaxEnt construction method to bivariate distributions.

\section{REFERENCES}

1. K. Pearson, "Contributions to the mathematical theory of evolution [abstract]", Proceedings of the Royal Society of London 54: 329-333.

2. K. Pearson, "Contributions to the mathematical theory of evolution, II: Skew variation in homogeneous material", Philosophical Transactions of the Royal Society of London 186: 343-414.

3. E. T. Jaynes, "Mathematical contributions to the theory of evolution, X: Supplement to a memoir on skew variation.", Philosophical Transactions of the Royal Society of London. Series A, Containing Papers of a Mathematical or Physical Character 197: 443-459. 
4. E. T. Jaynes, "Mathematical contributions to the theory of evolution, XIX: Second supplement to a memoir on skew variation.", Philosophical Transactions of the Royal Society of London. Series A, Containing Papers of a Mathematical or Physical Character 216: 429-457.

5. Wolfram Research, Inc. Champaign, IL, USA. http://www . wolfram. com

6. Pearson distribution. Retrieved May 23, 2011, from http://en.wikipedia.org/wiki/ Pearson_distribution

7. A. Rhind, "Tables to Facilitate the Computation of the Probable Errors of the Chief Constants of Skew Frequency Distributions", Biometrika 7(1/2): 127-147.

8. W. P. Elderton and N. L. Johnson, "Systems of frequency curves", Cambridge UK: Cambridge University Press

9. $\quad$ P. C. Gregory, "Bayesian Logical Data Analysis for the Physical Sciences.", New York: Cambridge University Press., edited by R. D. Levine and M. Tribus, MIT Press, 1979.

10. E. T. Jaynes, (G L Bretthorst, Ed.),"Probability Theory - The Logic of Science", Cambridge, UK: Cambridge University Press.

11. B. J. Stokes, "Continuous MaxEnt distributions in Mathematica: A "parameter-free" approach", in Bayesian Inference and Maximum Entropy Methods in Science and Engineering - 29th International Workshop, Oxford, Mississippi USA. (P M Goggans and C-Y Chan, Eds.). AIP Conference Proceedings 1193 American Institute of Physics. New York, pp 292-301. 
Copyright of AIP Conference Proceedings is the property of American Institute of Physics and its content may not be copied or emailed to multiple sites or posted to a listserv without the copyright holder's express written permission. However, users may print, download, or email articles for individual use. 